RODRIGO MELO GALINDO'

Frances Lanhellas Gonçalves'

RebeCA LoPES Figuera ${ }^{1}$

LOURENÇO SBRAGIA ${ }^{2}$

\section{Artigo de Revisão}

Palavras-chave

Hérnia diafragmática

Traqueia/fisiologia

Anormalidades congênitas/cirurgia

Keywords

Hernia, diaphragmatic Trachea/physiology

Congenital abnormalities/surgery

\title{
Manejo pré-natal da hérnia diafragmática congênita: presente, passado e futuro
}

\author{
Prenatal management of congenital diaphragmatic bernia: \\ present, past and future
}

\section{Resumo}

A hérnia diafragmática congênita é um defeito de formação do diafragma que acomete entre 1:2.000 e 1:4.000 nascidos vivos e constitui $8 \%$ das principais anomalias congênitas. Avanços médicos nos últimos 30 anos envolvendo diagnóstico pré-natal, intervenção fetal, manejo clinico e cirúrgico neonatal têm mudado a sobrevivência dos seus portadores. A evolução histórica desses avanços ajuda a compreender o esforço na busca de melhores resultados desse defeito muitas vezes fatal. Perspectivas na utilização de bioengenharia e terapia envolvendo células tronco podem trazer novas esperanças para os fetos com hérnia diafragmática congênita.

\section{Abstract}

The congenital diaphragmatic hernia is a defect in the formation of the diaphragm, which affects between $1: 2,000$ and $1: 4,000$ live births and represents $8 \%$ of major congenital anomalies. Medical advances in the last 30 years involving prenatal diagnosis, fetal intervention, neonatal surgical and clinical management have changed the survival of these patients. The historical evolution of these advances helps us to understand the effort in pursuit of better results of this defect, which is often lethal. Perspectives on the use of bioengineering and therapy involving stem cells may bring new hope for fetuses with congenital diaphragmatic hernia.

Correspondêncio

Lourenco Sbragia Divisũo de Cirurgia Pediátric Departamento de Cirurgia e Anatomio Faculdade de Medicina de Ribeirão Preto Universidade de São Paulo Avenida Bandeirantes, 3.900 - Monte Alegre CEP: 14049-900

Ribeirão Preto (SP), Brasil

Recebido $06 / 07 / 2014$

Aceito com modificaçōes

09/01/2015
Divisão de Cirurgia Pediátrica do Departamento de Cirurgia e Anatomia da Faculdade de Medicina de Ribeirão Preto da Universidade de São Paulo - USP - Ribeirão Preto (SP), Brasil.

'Laboratório de Cirurgia Fetal Experimental, Programa em Clínica Cirúrgica, Departamento de Cirurgia e Anatomia, Faculdade de Medicina de Ribeirão Preto, Universidade de São Paulo - USP - Ribeirão Preto (SP), Brasil.

${ }^{2}$ Divisão de Cirurgia Pediátrica, Departamento de Cirurgia e Anatomia, Faculdade de Medicina de Ribeirão Preto, Universidade de São Paulo - USP - Ribeirão Preto (SP), Brasil.

Conflito de interesses: não há. 
História

O primeiro relato de uma hérnia diafragmática congênita (HDC) foi feito em 1679 por Lazarus Riverius, em uma necrópsia de um paciente de 24 anos. Posteriormente, em 1761, Giovanni Battista Morgagni publicou uma revisão sobre hérnias diafragmáticas traumáticas e congênitas e descreveu a HDC anterior, que hoje leva seu nome. Victor Bochdalek, em 1848, descreveu pacientes com defeitos diafragmáticos póstero-laterais à direita e à esquerda. Defeitos nessa localização são hoje chamados de hérnia de Bochdalek e correspondem a $80 \%$ dos casos, sendo a apresentação mais comum de $\mathrm{HDC}^{1,2}$.

Realizamos um estudo de revisão histórica narrativa, realizado por meio de pesquisa na base de dados do PubMed utilizando os descritores: hérnia diafragmática congênita, tratamento, cirurgia fetal e oclusão traqueal, além de análise dos dados do Departamento de Informática do Sistema Único de Saúde (DATASUS), do Estudo Colaborativo Latino-Americano de Malformações Congênitas (ECLAMC) e da Organização Mundial de Saúde.

\section{Incidência}

A HDC acomete entre 1:2.000 e 1:4.000 nascidos vivos e constitui $8 \%$ das principais anomalias congêni$\operatorname{tas}^{1,3}$. O Atlas Mundial de Defeitos Congênitos (World Atlas of Birth Defects), publicação da Organização Mundial de Saúde, relata incidências entre 1:1.600, em Malta, e 1:23.800, na China ${ }^{4}$. Acomete predominantemente fetos do sexo masculino (1,5:1), mas não há diferença na incidência entre raças ${ }^{1}$. É mais frequente à esquerda $(80 \%)^{1}$ e cerca de $30 \%$ dos pacientes apresentam anomalias cromossômicas, tais como trissomia do 13 ou do 18 ou outros defeitos associados, sendo os mais frequentes os cardíacos ${ }^{5-7}$.

De acordo com dados do Departamento de Informática do Sistema Único de Saúde (DATASUS) foram registrados 1.075 nascimentos com HDC entre 2001 e 2009 no Sistema de Informações Sobre Nascidos Vivos (SINASC), o que corresponde a uma incidência aproximada de 1:25.000. No mesmo período, no Estado de São Paulo, a incidência foi de cerca de $1: 14.000^{8}$. Porém, esse número é muito subestimado, visto que nem sempre a HDC tem diagnóstico pré-natal ou é diagnosticada na sala de parto, momento em que a Declaração de Nascido Vivo é preenchida, pois o quadro clínico inicial se confunde com outras malformações, principalmente com as do aparelho respiratório e cardiovascular, podendo, inclusive, ser assintomática ou oligossintomática.

Dados do relatório final da $41^{\text {a }}$ Reunião Anual do Estudo Colaborativo Latino-Americano de Malformações
Congênitas (ECLAMC), em 2009, mostram uma incidência acumulada, entre os anos de 1982 e 2008, de 1:3.700 nos países e serviços participantes ${ }^{9}$. Essa incidência, bem maior do que a relatada pelo DATASUS, é superestimada, visto que foi colhida apenas em serviços de referência, porém, é mais próxima da incidência real devido ao rigor na coleta dos dados.

\section{Embriologia e fisiopatologia}

Embriologicamente, o diafragma é formado por quatro componentes: o septo transverso, o mesentério do esôfago, as membranas pleuro-peritoneais e a musculatura da parede do corpo ${ }^{10}$. Até a quinta semana de via embrionária, a cavidade torácica se comunica com a cavidade abdominal pelos canais pericárdio-peritoneais, que se fecham no fim da sexta semana com a fusão das membranas pleuro-peritoneais ${ }^{11}$. Na HDC ocorre um erro de sinalização celular que resulta no não fechamento dos canais pericárdio-peritoneais, com consequente passagem de órgãos abdominais para o interior da cavidade torácica ${ }^{11}$.

$\mathrm{Na}$ história natural da HDC, os órgãos abdominais presente dentro do tórax (geralmente fígado, estômago e intestino) atuam como massas que impedem que o pulmão cresça e acabam comprimindo o órgão e comprometendo seu desenvolvimento normal ${ }^{11}$. Essa compressão leva à hipoplasia pulmonar bilateral, pois há desvio do mediastino e também ocorre compressão do pulmão contralateral. Junto com a hipoplasia, há uma hipertrofia da camada média das arteríolas pulmonares e aumento da resistência vascular pulmonar, levando à hipertensão pulmonar (HP), que conforme sua gravidade pode levar à persistência do padrão fetal de circulação, acidose metabólica e respiratória, e morte neonatal ${ }^{3,12}$.

A hipoplasia pulmonar na HDC se caracteriza por um pulmão de tamanho menor do que o normal, diminuição da alveolarização, diminuição do volume alveolar, com diminuição da área de trocas gasosas, espessamento dos septos alveolares, alterações vasculares, como um aumento na espessura da camada média das arteríolas, e diminuição do conteúdo proteico e de DNA pulmonar bilateralmente $\mathrm{e}^{13,14}$.

\section{Manejo ventilatório neonatal}

A despeito do avanço nas unidades de cuidados intensivos neonatais, a mortalidade continuava alta até meados dos anos 1990. Mantinha-se em torno de 50\% para pacientes com diagnóstico isolado de HDC e em torno de $80 \%$ quando há anomalias associadas ${ }^{1,3,12,14}$. Harrison et al..$^{15}$ sugerem que a mortalidade perinatal seria ainda maior ao se considerar o óbito intrauterino 
de crianças sem o diagnóstico dessa anomalia (bidden mortality). O único relato de mortalidade por HDC em um serviço brasileiro foi publicado por Ruano et al. ${ }^{16}$, que avaliaram pacientes com diagnóstico pré-natal de HDC e encontraram uma mortalidade geral de $92 \%$ e de $89 \%$ nos portadores de diagnóstico isolado de HDC, todavia esses resultados podem não representar a real mortalidade brasileira.

Devido à hipoplasia e hipertensão pulmonares, os portadores de HDC são de difícil manuseio ventilatório, apresentando shunt direita-esquerda, hipóxia, hipercapnia e acidose mista. No intuito de aumentar a pressão parcial de oxigênio $\left(\mathrm{PaO}_{2}\right)$ e a saturação de oxigênio $\left(\mathrm{SatO}_{2}\right)$ e diminuir a pressão parcial de gás carbônico $\left(\mathrm{PaCO}_{2}\right)$, havia a tendência de aumentar a fração inspirada de oxigênio $\left(\mathrm{FiO}_{2}\right)$, aumentar a frequência ventilatória e aumentar os volumes ventilatórios, levando a pressões inspiratórias maiores. Com isso, a frequência de lesão pulmonar, barotrauma e pneumotórax nesses pacientes eram muito altas, o que muitas vezes causava o óbito.

O manejo da ventilação neonatal dos portadores de HDC vem sofrendo constantes modificações nas últimas décadas. Wung et al. ${ }^{17}$ instituíram o uso de baixas pressões ventilatórias, a hipercapnia permissiva, usando o mínimo possível de oxigênio suplementar (a chamada gentle ventilation), associado a uma postergação da correção cirúrgica da HDC. Observaram que a hipercapnia moderada, per se, não era nociva, desde que se pudesse corrigir a acidose farmacologicamente. Cronologicamente foi verificado que com o emprego da gentle ventitation o barotrauma e a lesão pulmonar foram minimizados ${ }^{3}$. A seguir, foi utilizado o ventilador de alta frequência (high frequency ventilator - HFV), que permitiu um maior recrutamento alveolar e a melhora da troca gasosa, diminuindo também o barotrauma. $\mathrm{O}$ uso de óxido nítrico inalatório (inhaled nitric oxideiNO), que leva a vasodilatação pulmonar e diminui o shunt direita-esquerda, também teve lugar no arsenal ventilatório, mas normalmente sabe-se que existe a necessidade de associação com drogas inotrópicas a fim de corrigir seu efeito colateral, que é a hipotensão sistêmica. Além do mais, sabe-se que o uso do iNO pode diminuir a indicação de oxigenação por membrana com circulação extracorpórea (extracorporeal membrane oxygenation - $\mathrm{ECMO})^{18}$. A colocação do paciente em ECMO permitiu a reversão da hipóxia, da hipercapnia e da acidose, podendo o pulmão permanecer em repouso por vários dias, o que pode diminuir a hipertensão local e restaurar a capacidade de trocas gasosas. No entanto, o real beneficio do ECMO ainda não está completamente comprovado na $\mathrm{HDC}^{19}$. Mais recentemente, em alguns centros, a mortalidade vem caindo para 20 a $30 \%$ com a instituição de protocolos de tratamento padronizados ${ }^{20,21}$.
Modelos animais para estudar hérnia diafragmática congênita

Os modelos animais de HDC são de fundamental importância para a compreensão dos mecanismos fisiopatogênicos da doença e suas complicações, dos mecanismos genéticos e moleculares, além de avaliar possíveis tratamentos pré- e pós-natais. Os três tipos principais são os cirúrgicos, em vários animais como ovelhas ${ }^{22}$, $\operatorname{coelhos}^{23}$ e ratos ${ }^{24}$, os genéticos e os teratogênicos ${ }^{25,26}$. O mais utilizado é o toxicológico em ratos com o uso do Nitrofen (2,4-dicloro-4'nitrodifenil éter). O Nitrofen é um teratógeno que quando administrado no dia gestacional (DG) 9,5, na dose de $100 \mathrm{mg}$, causa HDC em cerca de $40 \%$ dos fetos de ratas ${ }^{27}$.

O modelo de HDC em ratos tem várias vantagens: curto período gestacional (termo corresponde ao $22^{\circ}$ dia de gestação), maior número de fetos por gestação, baixo custo de aquisição e manutenção, além de possibilitar uma vasta gama de estudos de biologia molecular, não disponíveis em animais de maior porte ${ }^{28}$.

\section{História da intervenção fetal e o diagnóstico pré-natal}

Estudos experimentais, nos quais o conceito de correção fetal de um defeito pudesse minimizar os efeitos neonatais da mesma doença, abriram caminho para o desenvolvimento das intervenções fetais de várias anomalias congênitas. Historicamente, a correção cirúrgica fetal da HDC sofreu várias etapas e estratégias no intuito de minimizar os efeitos da hipertensão pulmonar.

A HDC foi um dos primeiros defeitos a serem considerados para o racional tratamento da correção fetal, ou seja, a correção pré-natal minimizaria ou mesmo cessaria a evolução da hipoplasia pulmonar e suas consequências. Harrison et al. ${ }^{12}$, na University of California, San Francisco (UCSF), começaram um ensaio clínico no qual foi empregada a correção cirúrgica fetal da HDC. Os resultados iniciais, de 1990, concluíram que quando havia herniação do fígado dentro do tórax, a correção fetal não deveria ser realizada, pois com a redução do fígado para a cavidade abdominal havia um acotovelamento da veia umbilical e consequente óbito fetal ${ }^{12}$.

O mesmo grupo conduziu um novo ensaio para correção cirúrgica de fetos com HDC, no período de 1993 a 1996, mas sem evidência de componente hepático no tórax. Nesse segundo estudo, não foi observada diferença na sobrevida entre os pacientes operados no período fetal e os tratados convencionalmente com cirurgia neonatal. Os autores concluíram que não há indicação de correção fetal da hérnia nos pacientes com HDC sem herniação do fígado para o tórax, pois esses pacientes têm um 
prognóstico melhor, independente do tratamento, além do risco potencial da cirurgia para o feto e para a mãe ${ }^{29}$.

A obstrução congênita das vias aéreas superiores é uma anomalia muito rara. Hedrick et al. ${ }^{30}$ descreveram uma série de quatro casos diagnosticados durante o pré-natal e criaram o termo obstrução congênita das vias aéreas superiores (congenital high airway obstruction syndrome CHAOS), em que todos os fetos apresentavam achados similares: grandes pulmões ecogênicos, diafragmas retificados ou invertidos, vias aéreas dilatadas distalmente à obstrução e ascite ou hidrópsia fetal ${ }^{30}$.

Com base nesses achados, DiFiore et al. ${ }^{22}$ criaram um modelo de tráqueo-oclusão (TO) em fetos de ovelhas com HDC no intuito de promover o crescimento do pulmão intraútero e reverter a hipoplasia pulmonar ${ }^{12}$. Os resultados demonstraram que a TO impedia a saída de fluido produzido normalmente pelo pulmão e, por ação mecânica, levava à distensão alveolar, crescimento e aceleração da maturidade e desenvolvimento da vasculatura pulmonar. Essas mudanças reduziam os efeitos deletérios da hipoplasia e da hipertensão pulmonar ${ }^{12,22,24}$.

A TO aumenta gradualmente a pressão intratraqueal e leva à expansão do pulmão, principal fator responsável pela aceleração do seu crescimento. Após a retirada da $\mathrm{TO}$, ocorre um grande aumento da quantidade de muco intrapulmonar, que não está esclarecido se é devido ao acúmulo que ocorreu em consequência da TO ou ao aumento da produção de muco pelas células caliciformes do epitélio traqueal ${ }^{24,31,32}$.

Harrison et al. ${ }^{31}$ avaliaram fetos que não entraram no ensaio clínico de correção cirúrgica fetal da HDC por terem herniação hepática intratorácica e iniciaram um protocolo de TO fetal por via aberta, ou seja, por histerotomia materna, para acessar a traqueia fetal. Inicialmente a oclusão foi realizada com um tampão esponjoso intratraqueal e posteriormente com um clipe extratraqueal. Para a retirada do clipe foi necessário desenvolver uma estratégia para ter acesso à via respiratória do feto denominada ex-utero intrapartum treatment (EXIT). EXIT é definido pela retirada da oclusão traqueal por meio de cesariana, quando o polo cefálico fetal é exteriorizado na incisão, mantendo a circulação placentária até a retirada da TO e intubação traqueal, seguido da retirada do feto e clampeamento do cordão fetal assim que a via respiratória estiver garantida pela intubação ${ }^{31}$. Posteriormente, essa técnica passou a ser usada para acessar a via traqueal em fetos com massas cervicais gigantes ${ }^{33}$.

Os resultados iniciais da TO com clipe foram publicados em 1996 e os finais em 2000, com a conclusão de que a técnica promovia o crescimento pulmonar, mas a mortalidade e as complicações eram muito grandes, em especial, prematuridade ${ }^{31,34}$. Ainda em 1996, VanderWall et al. ${ }^{35}$, também da UCSF, publicaram o aprimoramento da técnica de clipagem traqueal, substituindo o acesso fetal por cirurgia materna com abertura uterina, pelo acesso fechado por meio da utilização de fetoscopia videoassistida. O procedimento foi denominado, Fetendo-Clip ${ }^{35}$, intervenção bem menos invasiva, que foi rapidamente empregada na pratica clínica ${ }^{36}$. Embora tenha tido avanço na via de acesso, essa técnica também foi abandonada devido às altas taxas de prematuridade e de lesões irreversíveis que ocorriam na traqueia e no nervo laríngeo recorrente dos fetos ${ }^{37}$.

Benachi et al. ${ }^{38}$ descreveram uma técnica menos invasiva de TO, em fetos de ovelhas, usando um balão destacável também por via fetoscópica videoassistida. O grupo da UCSF, Harrison et al. ${ }^{39}$, relatou dois pacientes em que realizaram a TO utilizando um trocáter de $5 \mathrm{~mm}$, para acesso ao útero, e um histeroscópio reto de $4,5 \mathrm{~mm}$ para aposição do balão. $\mathrm{O}$ balão foi removido por EXIT $^{39}$. O mesmo grupo publicou ${ }^{40}$ os resultados de um ensaio clínico randomizado comparando o tratamento endoscópico colocando o balão fetal e o convencional operando a HDC no período neonatal. Esse estudo foi interrompido precocemente, pois os resultados do tratamento endoscópico não foram superiores ao tratamento convencional da correção da HDC devido a altas taxas de prematuridade, média de 30,8 $\pm 2,0$ semanas de idade gestacional, e de rotura prematura das membranas que atingiu $100 \%$.

Concomitante ao avanço das técnicas de intervenção fetal houve também avanço na qualificação da ultrassonografia fetal com desenvolvimento de marcadores prognósticos da HDC. Entre os marcadores prognósticos, os mais importantes são a presença de herniação hepática (liver up) e o índice pulmão/cabeça (lung-to-head ratio - LHR). A presença do fígado intratorácico leva a um maior grau de hipoplasia e tem relação direta com a sobrevivência ${ }^{3,29,41}$.

O LHR, um indicador ultrassonográfico de sobrevida, foi descrito em 1996 por Metkus et al. ${ }^{42}$ apenas em pacientes com HDC à esquerda. O cálculo desse marcador era feito dividindo a área do pulmão direito, aferido em um corte transverso do tórax fetal, na altura do átrio direito, pelo perímetro cefálico do feto. A área do pulmão era calculada multiplicando os maiores diâmetros perpendiculares do pulmão, em milímetros. Usava-se o pulmão direito, pois nem sempre é possível individualizar o pulmão esquerdo do conteúdo herniário. O perímetro cefálico era usado para diminuir diferenças pulmonares devido à idade gestacional ${ }^{42}$. No entanto, as imagens utilizadas para explicar a técnica eram inconsistentes, levando a resultados diferentes nos estudos posteriores e à criação de outras técnicas de medição ${ }^{41}$.

Atualmente, o método mais utilizado e que melhor mede o grau de hipoplasia pulmonar é o LHR aferido 
na altura das quatro câmaras cardíacas, levando-se em consideração o produto dos maiores diâmetros do pulmão contralateral à hérnia, possibilitando medir o LHR quando a HDC se apresenta à direita ${ }^{43}$. Geralmente, fetos com LHR abaixo de 1,0 têm prognóstico muito ruim, de 0 a $20 \%$ de sobrevivência; pacientes com LHR entre 1,0 e 1,4 têm prognóstico intermediário, de 38 a $60 \%$ de sobrevivência e acima de 1,4 têm bom prognóstico, de 73 a $100 \%$ de sobrevivência ${ }^{44}$.

Trabalhos mais recentes têm apontado que o indicador prognóstico mais fidedigno é a utilização do LHR observado/esperado (o/e LHR), independente da presença ou não de fígado no tórax, da localização da hérnia ou da idade gestacional ${ }^{41}$. Descrito por Jani et al. ${ }^{44}$, o o/e LHR é o resultado da divisão do LHR no feto com HDC pelo LHR de fetos normais na mesma idade gestacional.

Papadakis et al. ${ }^{45}$ publicaram estudo em fetos de ovelhas, nos quais realizaram a TO utilizando um trocáter de $5 \mathrm{~mm}$, uma bainha de 9 Fr e um fetoscópio, sendo os dois últimos mais finos (3,0 e 1,2 mm, respectivamente) do que aquele utilizado por Harrison et al. ${ }^{39}$. Deprest et al ${ }^{46}$ aperfeiçoaram a técnica minimamente invasiva, usando apenas a bainha e o fetoscópio para acesso ao útero, e fazendo a retirada do balão cerca de 15 dias após o primeiro procedimento. O mesmo grupo publicou ${ }^{7}$ os resultados dos primeiros 21 casos dessa nova técnica denominada oclusão traqueal fetoscópica (fetoscopic tracheal occlusion - FETO), usando o equipamento descrito. FETO é indicado em pacientes sem malformações associadas ou anomalias cromossômicas, com HDC de mal prognóstico, i.e., com herniação hepática (liver up) e LHR abaixo de 1,0. Nessa nova técnica foi observada uma taxa de $52 \%$ de amniorrexe prematura e $90,5 \%$ de partos prematuros, média de $33,3 \pm 3,3$ semanas de idade gestacional ${ }^{7}$, médias bem menores do que as relatadas pelo grupo da UCSF que usou um trocáter de $5 \mathrm{~mm}^{40}$. No estudo multicêntrico europeu realizado por Jani et al. ${ }^{47}$ foram avaliados 210 casos submetidos à FETO e foi verificado um aumento do LHR e, consequentemente, da sobrevida para $48 \%$, com $47,1 \%$ de amniorrexe prematura e média de 35,3 semanas de gestação.

Hoje, sabe-se que a idade gestacional ideal para e realização da FETO é entre 26 e 29 semanas, no fim da fase canalicular do desenvolvimento pulmonar. O balão deve ser retirado com 34 semanas após a aposição, pois um tempo prolongado de oclusão traqueal pode lesar os pneumócitos tipo II e levar à diminuição da produção de surfactante ${ }^{47-49}$.

Embora a utilização da FETO seja um notável avanço no aumento da sobrevida de fetos com HDC grave, sabe-se que o balão causa leves alterações na traqueia, com mudanças inflamatórias locais, e defeitos epiteliais limitados, como diminuição da superfície de contato com o $a r^{46}$. Contudo, essas modificações ainda não foram morfologicamente bem estudadas, nem sua evolução temporal. Radiologicamente foi observada traqueomegalia em lactentes que sobreviveram e tinham sido submetidos à FETO, porém, sem impacto clínico importante além de uma tosse ladrante ${ }^{49-51}$. É possível que os efeitos da TO, tanto benéficos como adversos, não se restrinjam apenas aos pulmões e possam se estender à traqueia ${ }^{32}$.

A despeito do grande avanço na compreensão do desenvolvimento da história natural e no tratamento perinatal da HDC nos últimos 30 anos, ainda não há um completo consenso no benefício empregando FETO. Há uma discordância entre americanos e europeus (Eurofetus). Alguns autores norte-americanos advogam que não existe um marcador pré-natal específico para o prognóstico e mesmo a evolução da história natural da doença é profundamente variável e dependente de uma série de fatores que vai do correto diagnóstico pré-natal ao atendimento pós-natal, fatores esses que influenciam dramaticamente a evolução, especialmente se o centro de atendimento neonatal for terciário e com experiência no manejo da $\mathrm{HDC}^{52}$. Isso estaria demonstrado pela falha da maioria dos estudos tentando correlacionar os fatores pré e pós-natais da HDC, mesmo nos ensaios clínicos. A experiência com TO em dois centros terciários de referência nos EUA mostrou melhora na sobrevivência muito mais devido ao cuidado neonatal ${ }^{40}$, que envolveu enormes custos econômicos e sequelas clínicas, do que à intervenção fetal propriamente realizada ${ }^{34,40}$. No momento, dois novos ensaios clínicos promovidos pelo Eurofetus envolvendo o prognóstico e a estratificação da FETO para HDC moderada e grave, especialmente aquelas que envolvem a colocação de patch para a reconstrução do diafragma, talvez possam esclarecer essas dúvidas e provar ainda mais sua eficácia para os grupos americanos.

\section{FETO no Brasil}

Peralta et al..$^{53}$, em 2011, compararam a sobrevivência pós-natal de 28 fetos com HDC esquerda grave e isolada que foram submetidos à FETO com a de 13 fetos controles não randomizados no período de quatro anos e concluíram que houve melhora significativa na sobrevida pós-natal com alta do berçário (de 0 para 35,6\%) de fetos com HDC.

Ruano et al. ${ }^{54}$, em 2012, realizaram o mesmo estudo randomizado e controlado para avaliar o efeito da FETO entre 20 fetos com HDC grave e 21 controles que foram submetidos à cirurgia neonatal. A diferença desse estudo em relação a outros estudos foi que a remoção do balão foi feita por EXIT ao nascimento ao invés de se retirar o balão antes do nascimento como recomendam os autores da FETO $^{49}$. Os autores obtiveram uma maior taxa de sobrevivência entre o grupo FETO em relação ao grupo controle $(50 \text { versus } 4,8 \%)^{54}$. 
Embora essas experiências pioneiras em tratamento fetal da HDC sejam promissoras, por razões políticas e econômicas ainda estamos muito aquém do suporte completo e adequado pós-natal da doença, especialmente pela carência de um centro de referência neonatal especializado onde se tenha ECMO com um protocolo adequado à doença a ser implantado no país.

\section{Perspectivas}

Algumas perspectivas, além dos ensaios clínicos, podem ser consideradas promissoras. A colocação do balão fetal por FETO mais precoce (anterior às 24 semanas) para os fetos de muito mal prognóstico $(\mathrm{LHR}<0,7)$ talvez possa fazer com que haja um maior crescimento pulmonar, pois ainda não está comprovado se ocorre uma efetiva melhora da sobrevida ${ }^{55}$.

O crescimento da medicina regenerativa, da bioengenharia e da terapia celular nas últimas décadas também ocupou espaço nas perspectivas de tratamento da HDC. Turner et al. ${ }^{56}$ validou a utilização de matriz para o implante de células tronco mesenquimais do líquido amniótico em ovelhas com a finalidade de criar um diafragma a partir de células autólogas. Fauza ${ }^{57}$, em artigo de revisão, comenta a retirada das mesmas células de humanos e a implantação em uma matriz acelular construindo um "músculo diafragma" a partir das próprias células do paciente. Evidentemente, isso não é uma prática corriqueira, uma vez que o Food and Drug Administration (FDA) ainda não permite sua utilização, mas é possível construir um tendão muscular diafragmático autólogo, sem a necessidade de colocar uma prótese nas situações graves quando não existe diafragma ${ }^{57}$, visto que o uso de próteses pode levar a uma taxa de recidiva da hérnia de até $50 \%{ }^{58}$.
Futuramente, o mesmo tratamento pode ser empregado para melhorar não só o crescimento, mas também a hipertensão pulmonar quando ocorre uma reestruturação do parênquima a partir de células tronco já identificadas no próprio pulmão $0^{59}$. No modelo experimental de lesão pulmonar em ratos, a utilização de células tronco de origem pulmonar formou bronquíolos e alvéolos, e houve remodelação pulmonar ${ }^{60}$. Esses dados suportam a ideia de que possa haver a capacidade de tratamento para a hipoplasia e a hipertensão pulmonar na HDC.

\section{Conclusão}

Avanços no diagnóstico pré-natal, no aconselhamento familiar e na possibilidade de tratamento da HDC grave antes do nascimento são realidades que podem aumentar a sobrevivência de fetos portadores desse defeito. A utilização de bioengenharia associada à terapia com células tronco para reconstrução diafragmática é factível e pode ser empregada nos fetos com agenesia de diafragma. No Brasil, há necessidade de um maior alinhamento e organização entre as equipes multidisciplinares a fim de oferecer todo o suporte necessário que envolve o acompanhamento neonatal desses pacientes, especialmente no que se refere ao emprego de ECMO na HDC.

\section{Agradecimentos}

Os autores agradecem à Fundação de Amparo à Pesquisa do Estado de São Paulo (FAPESP - Bolsa de Investigação $n^{0}$ 11/00794-1) e à Coordenação de Aperfeiçoamento de Pessoal de Nível Superior (CAPES) pelos apoios financeiros.

\section{Referências}

1. Doyle NM, Lally KP. The CDH Study Group and advances in the clinical care of the patient with congenital diaphragmatic hernia. Semin Perinatol. 2004;28(3):174-84.

2. Greer JJ. Current concepts on the pathogenesis and etiology of congenital diaphragmatic hernia. Respir Physiol Neurobiol. 2013;189(2):232-40

3. Laberge $\mathrm{JM}$, Flageole $\mathrm{H}$. Fetal tracheal occlusion for the treatment of congenital diaphragmatic hernia. World J Surg. 2007;31 (8):1577-86.

4. World Health Organization (WHO). World atlas of birth defects [Internet]. Geneva: WHO; 2003 [cited 2014 May 22]. Available from: <http://www.who.int/genomics/about/en/diaphragmatic. pdf? ua $=1>$

5. Tonks A, Wyldes M, Somerset DA, Dent K, Abhyankar A, Bagchi $\mathrm{I}$, et al. Congenital malformations of the diaphragm: findings of the West Midlands Congenital Anomaly Register 1995 to 2000. Prenat Diagn. 2004;24(8):596-604.
6. Braby J. Current and emerging treatment for congenital diaphragmatic hernia. Neonatal Netw. 2001;20(2):5-15.

7. Deprest J, Gratacos E, Nicolaides KH, FETO Task Group. Fetoscopic tracheal occlusion (FETO) for severe congenital diaphragmatic hernia: evolution of a technique and preliminary results. Ultrasound Obstet Gynecol. 2004;24(2):121-6.

8. Brasil. Ministério da Saúde. DATASUS. Informações de Saúde. Nascidos vivos. Download de arquivos - a partir de 1996 [Internet]. Brasília: Ministério da Saúde; 2014 [citado 2014 Maio 22]. Disponível em: <http://tabnet.datasus.gov.br/cgi/sinasc/dados/ nov_indice.htm>

9. Estudio Colaborativo Latino Americano de Malformaciones Congénitas [Internet]. XXXXI Reunion Anual del Estudio Colaborativo Latinoamericano de Malformaciones Congenitas. Angra dos Reis; 2009 [citado 2014 May 23]. Disponível em: <http://www. eclamc.org/descargas/DF-RAE4 1_Parte\%201.pdf> 
10. Moore KL, Persaud TVN, Torchia MG. Embriologia clínica. 8a ed. Rio de Janeiro: Elsevier; 2008.

11. Mayer S, Metzger R, Kluth D. The embryology of the diaphragm. Semin Pediatr Surg. $2011 ; 20(3): 161-9$.

12. Harrison MR, Langer JC, Adzick NS, Golbus MS, Filly RA, Anderson $\mathrm{RL}$, et al. Correction of congenital diaphragmatic hernia in utero, V. Initial clinical experience. J Pediatr Surg. 1990;25(1):47-55.

13. Kotecha S, Barbato A, Bush A, Claus F, Davenport M, Delacourt $C$, et al. Congenital diaphragmatic hernia. Eur Respir J. 2012;39(4):820-9.

14. Deprest JA, Gratacos E, Nicolaides K, Done E, Van Mieghem $T$, Gucciardo L, et al. Changing perspectives on the perinatal management of isolated congenital diaphragmatic hernia in Europe. Clin Perinatol. 2009;36(2):329-47.

15. Harrison MR, Adzick NS, Estes JM, Howell L. A prospective study of the outcome for fetuses with diaphragmatic hernia. JAMA. $1994 ; 271(5): 382-4$

16. Ruano R, Bunduki V, Silva MM, Yoshizaki CT, Tanuri U, Macksoud $J G$, et al. Prenatal diagnosis and perinatal outcome of 38 cases with congenital diaphragmatic hernia: 8-year experience of a tertiary Brazilian center. Clinics (Sao Paulo). 2006;61(3):197-202.

17. Wung JT, Sahni R, Moffitt ST, Lipsitz E, Stolar CJ. Congenital diaphragmatic hernia: survival treated with very delayed surgery, spontaneous respiration, and no chest tube. J Pediatr Surg. 1995;30(3):406-9.

18. Neonatal Inhaled Nitric Oxide Study Group (NINOS). Inhaled nitric oxide and hypoxic respiratory failure in infants with congenital diaphragmatic hernia. Pediatrics. 1997;99(6):838-45.

19. Mugford M, Elbourne D, Field D. Extracorporeal membrane oxygenation for severe respiratory failure in newborn infants. Cochrane Database Syst Rev. 2008;3:CD001340.

20. Rollins MD. Recent advances in the management of congenital diaphragmatic hernia. Curr Opin Pediatr. 2012;24(3):379-85.

21. van den Hout L, Schaible T, Cohen-Overbeek TE, Hop W, Siemer $\mathrm{J}$, van de Ven K, et al. Actual outcome in infants with congenital diaphragmatic hernia: the role of a standardized postnatal treatment protocol. Fetal Diagn Ther. $2011 ; 29(1): 55-63$

22. DiFiore JW, Fauza DO, Slavin R, Peters CA, Fackler JC, Wilson $J M$. Experimental fetal tracheal ligation reverses the structural and physiological effects of pulmonary hypoplasia in congenital diaphragmatic hernia. J Pediatr Surg. 1994;29(2):248-56.

23. De Paepe ME, Johnson BD, Papadakis K, Sueishi K, Luks Fl. Temporal pattern of accelerated lung growth after tracheal occlusion in the fetal rabbit. Am J Pathol. 1998; 152(1):179-90.

24. Kitano Y, Yang EY, von Allmen D, Quinn TM, Adzick NS, Flake AW. Tracheal occlusion in the fetal rat: a new experimental model for the study of accelerated lung growth. J Pediatr Surg. 1998;33(12):1741-4.

25. Beurskens $N$, Klaassens $M$, Rottier R, de Klein A, Tibboel D. Linking animal models to human congenital diaphragmatic hernia. Birth Defects Res A Clin Mol Teratol. 2007;79(8):565-72.

26. van Loenhout RB, Tibboel D, Post M, Keijzer R. Congenital diaphragmatic hernia: comparison of animal models and relevance to the human situation. Neonatology. 2009;96(3):137-49.

27. Kluth $D$, Kangah $R$, Reich $P$, Tenbrinck R, Tibboel D, Lambrecht $W$. Nitrofen-induced diaphragmatic hernias in rats: an animal model. J Pediatr Surg. 1990;25(8):850-4

28. Kitano $Y$, Kanai M, Davies $P$, von Allmen D, Yang EY, Radu A, et al. BAPS prize-1999: lung growth induced by prenatal tracheal occlusion and its modifying factors: a study in the rat model of congenital diaphragmatic hernia. J Pediatr Surg. 2001;36(2):251-9.
29. Harrison MR, Adzick NS, Bullard KM, Farrell JA, Howell L, Rosen $M A$, et al. Correction of congenital diaphragmatic hernia in utero VII: a prospective trial. J Pediatr Surg. 1997;32(11):1637-42.

30. Hedrick MH, Ferro MM, Filly RA, Flake AW, Harrison MR, Adzick NS. Congenital high airway obstruction syndrome (CHAOS): a potential for perinatal intervention. J Pediatr Surg. $1994 ; 29(2): 271-4$

31. Harrison MR, Adzick NS, Flake AW, VanderWall KJ, Bealer JF, Howell $\amalg$, et al. Correction of congenital diaphragmatic hernia in utero VIII: response of the hypoplastic lung to tracheal occlusion. J Pediatr Surg. 1996;31(10):1339-48.

32. Gallindo RM, Gonçalves FL, Barreto CT, Schmidt AF, Pereira LA, Sbragia L. Evaluation of histological changes after tracheal occlusion at different gestational ages in a fetal rat model. Clinics (Sao Paulo). 2013;68(1):59-63.

33. Liechty KW, Crombleholme TM, Flake AW, Morgan MA, Kurth $C D$, Hubbard $A M$, et al. Intrapartum airway management for giant fetal neck masses: the EXIT (ex utero intrapartum treatment) procedure. Am J Obstet Gynecol. 1997;177(4):870-4.

34. Flake AW, Crombleholme TM, Johnson MP, Howell U, Adzick NS. Treatment of severe congenital diaphragmatic hernia by fetal tracheal occlusion: clinical experience with fifteen cases. Am J Obstet Gynecol. 2000; 183(5):1059-66.

35. VanderWall KJ, Bruch SW, Meuli M, Kohl T, Szabo Z, Adzick NS, et al. Fetal endoscopic ('Fetendo') tracheal clip. J Pediatr Surg. 1996;31(8):1101-3.

36. VanderWall KJ, Skarsgard ED, Filly RA, Eckert J, Harrison MR. Fetendo-clip: a fetal endoscopic tracheal clip procedure in a human fetus. J Pediatr Surg. 1997;32(7):970-2.

37. Harrison MR, Sydorak RM, Farrell JA, Kitterman JA, Filly RA, Albanese CT. Fetoscopic temporary tracheal occlusion for congenital diaphragmatic hernia: prelude to a randomized, controlled trial. J Pediatr Surg. 2003;38(7):1012-20.

38. Benachi A, Dommergues M, Delezoide AL, Bourbon J, Dumez Y, Brunnelle F. Tracheal obstruction in experimental diaphragmatic hernia: an endoscopic approach in the fetal lamb. Prenat Diagn. 1997; 17(7):629-34.

39. Harrison MR, Albanese CT, Hawgood SB, Farmer DL, Farrell $\mathrm{JA}$, Sandberg PL, et al. Fetoscopic temporary tracheal occlusion by means of detachable balloon for congenital diaphragmatic hernia. Am J Obstet Gynecol. 2001;185(3):730-3.

40. Harrison MR, Keller RL, Hawgood SB, Kitterman JA, Sandberg PL, Farmer DL, et al. A randomized trial of fetal endoscopic tracheal occlusion for severe fetal congenital diaphragmatic hernia. $\mathrm{N}$ Engl J Med. 2003;349(20):1916-24.

41. Jani JC, Peralta CF, Nicolaides KH. Lung-to-head ratio: a need to unify the technique. Ultrasound Obstet Gynecol. $2012 ; 39(1): 2-6$

42. Metkus AP, Filly RA, Stringer MD, Harrison MR, Adzick NS. Sonographic predictors of survival in fetal diaphragmatic hernia. J Pediatr Surg. 1996;31(1):148-51

43. Doné E, Gucciardo L, Van Mieghem T, Jani J, Cannie M, Van Schoubroeck D, et al. Prenatal diagnosis, prediction of outcome and in utero therapy of isolated congenital diaphragmatic hernia. Prenat Diagn. 2008;28(7):581-91.

44. Jani J, Keller RL, Benachi A, Nicolaides KH, Favre R, Gratacos $E$, et al. Prenatal prediction of survival in isolated left-sided diaphragmatic hernia. Ultrasound Obstet Gynecol. $2006 ; 27(1): 18-22$

45. Papadakis K, Luks Fl, Deprest JA, Evrard VE, Flageole H, Miserez $M$, et al. Single-port tracheoscopic surgery in the fetal lamb. J Pediatr Surg. 1998;33(6):918-20. 
46. Deprest JA, Evrard VA, Verbeken EK, Perales AJ, Delaere PR, Lerut $T E$, et al. Tracheal side effects of endoscopic balloon tracheal occlusion in the fetal lamb model. Eur J Obstet Gynecol Reprod Biol. 2000;92(1): 119-26.

47. Jani JC, Nicolaides KH, Gratacós E, Valencia CM, Doné E, Martinez JM, et al. Severe diaphragmatic hernia treated by fetal endoscopic tracheal occlusion. Ultrasound Obstet Gynecol. 2009;34(3):304-10.

48. Deprest J, Jani J, Gratacos E, Vandecruys H, Naulaers G, Delgado J, et al. Fetal intervention for congenital diaphragmatic hernia: the European experience. Semin Perinatol. 2005;29(2):94-103.

49. Deprest J, Nicolaides K, Done' E, Lewi P, Barki G, Largen E, et al. Technical aspects of fetal endoscopic tracheal occlusion for congenital diaphragmatic hernia. J Pediatr Surg. 201 1;46(1):22-32.

50. Jani J, Valencia C, Cannie M, Vuckovic A, Sellars M, Nicolaides $\mathrm{KH}$. Tracheal diameter at birth in severe congenital diaphragmatic hernia treated by fetal endoscopic tracheal occlusion. Prenat Diagn. $2011 ; 31$ (7):699-704.

51. Deprest J, Breysem L, Gratacos E, Nicolaides K, Claus F, Debeer A, et al. Tracheal side effects following fetal endoscopic tracheal occlusion for severe congenital diaphragmatic hernia. Pediatr Radiol. 2010;40(5):670-3.

52. Vrecenak JD, Flake AW. Fetal surgical intervention: progress and perspectives. Pediatr Surg Int. 2013;29(5):407-17.

53. Peralta CF, Sbragia L, Bennini JR, Cavalli RC, Rousselet MS, Barini R. [Tracheal occlusion for fetuses with severe isolated left-sided diaphragmatic hernia: a nonrandomized controlled experimental study]. Rev Bras Ginecol Obstet. 2011 ;33(12):381-7. Portuguese.

54. Ruano R, Yoshisaki CT, da Silva MM, Ceccon ME, Grasi MS, Tannuri $U$, et al. A randomized controlled trial of fetal endoscopic tracheal occlusion versus postnatal management of severe isolated congenital diaphragmatic hernia. Ultrasound Obstet Gynecol. 2012;39(1):20-7.

55. Ruano R, Peiro JL, da Silva MM, Campos JA, Carreras E, Tannuri $U$, et al. Early fetoscopic tracheal occlusion for extremely severe pulmonary hypoplasia in isolated congenital diaphragmatic hernia: preliminary results. Ultrasound Obstet Gynecol. 2013;42(1):70-6.

56. Turner CG, Klein JD, Steigman SA, Armant M, Nicksa GA, Zurakowski $D$, et al. Preclinical regulatory validation of an engineered diaphragmatic tendon made with amniotic mesenchymal stem cells. J Pediatr Surg. 201 1;46(1):57-61.

57. Fauza DO. Tissue engineering in congenital diaphragmatic hernia. Semin Pediatr Surg. 2014;23(3):135-40.

58. Tsai J, Sulkowsky J, Adzick NS, Hedrick HL, Flake AW. Patch repair for congenital diaphragmatic hernia: is it really a problem? J Pediatr Surg. 2012;47(4):637-41.

59. Anversa P, Kajstura J, Leri A, Loscalzo J. Tissue-specific adult stem cells in the human lung. Nat Med. $2011 ; 17(9): 1038-9$.

60. Kajstura J, Rota M, Hall SR, Hosoda T, D'Amario D, Sanada $F$, et al. Evidence for human lung stem cells. $N$ Engl J Med. $2011 ; 364(19): 1795-806$. 